\title{
GALOIS THEORY OF DIFFERENTIAL FIELDS OF POSITIVE CHARACTERISTIC
}

\author{
Kayoko ShIKISHIMA-TsujI
}

\begin{abstract}
The strongly normal extensions of a differential field $K$ of positive characteristic are defined. On the set $G$ of all differential isomorphisms of a strongly normal extension $N$ of $K$, a structure of an algebraic group is induced. Correspondences between subgroups of $G$ and intermediate differential fields of $N$ and $K$ are studied.
\end{abstract}

0. Introduction. E. R. Kolchin established a Galois theory for differential fields of characteristic zero (Kolchin [1]).

Galois groups of Picard-Vessiot extensions are algebraic matrix groups, and those of strongly normal extensions are algebraic groups. As general algebraic groups are well studied for arbitrary characteristic, it has been quite desirable to develop a Galois theory for differential fields of positive characteristic to the level of Kolchin's work in characteristic zero.

For differential fields of positive characteristic, however, such a theory has been known only for Picard-Vessiot extensions, with the field of constants of the ground differential field algebraically closed.

The purpose of the present work is to develop a theory of strongly normal extensions, which are more general than Picard-Vessiot extensions. This permits us to construct the theory of Picard-Vessiot extensions without restrictions and also to investigate more in detail the properties of Picard-Vessiot extensions (Okugawa [2]).

To meet this purpose, we had to develop many basic properties of differential fields of positive characteristic. The results of these works have been already published elsewhere ([2], Shikishima [4] and Tsuji (nee Shikishima) [5]).

As has been done by K. Okugawa [3], we adopt in this work the commutative and iterative higher derivations of infinite rank, which are more natural than the usual derivations. Under the usual derivations, when the characteristic $p$ is positive, the $p$ th power of any element in a differential field is a constant. This creates various irregularities. For example in this case, there is no proper strongly normal extension of a differential field. 
This paper has five sections. The conventions used throughout are explained in $\S 1$. In $\S 2$, we state some preliminary results on differential isomorphisms. In $\S 3$, strongly normal differential extensions and their Galois groups are studied. $\S 4$ is devoted to the fundamental theorems of Galois theory. Finally, we provide two examples in $\S 5$.

1. Conventions. Throughout this paper, we denote a differential field of positive characteristic $p$ by $K$, and also a differential extension field of $K$ by $N$. Let $\Delta$ be the set of commutative and iterative higher derivation operators of infinite rank $\delta_{i}=\left(\delta_{i \nu} ; \nu=0,1, \ldots\right)(i \in I)$, where $I$ is the set of indices. The set of derivative operators, namely, the set of all products of finitely many distinct members of $\left\{\delta_{i \nu} ; i \in I\right.$, $\nu \geq 0\}$, is denoted by $\Theta$. The algebraic closure of $K$ in $N$ is represented by $K^{0}$ and the field of constants of $N$ by $C$.

For a given field $M$, we denote by $M_{a}$ an algebraic closure of $M$, by $M_{s}$ the separable closure of $M$ in $M_{a}$, and by $M_{i}$ the purely inseparable closure of $M$ in $M_{a}$. In the case where $M$ is a differential extension of $K$, the differential closure of $M$ in $M_{a}$, that is, the largest differential extension of $M$ in $M_{a}$, is denoted by $M_{\Delta}$ (see [4] or [5]). The field of constants of $M$ is denoted by $M_{c}$.

We fix once and for all a universal differential extension $U$ of $N_{\Delta}$ (whence, of $K$ ) (see [4] and [2]). Whenever differential isomorphisms of a differential field $M$ are considered, they are tacitly assumed to be "into $U$ ".

We mean the $e$ th power of the characteristic $p$ by $p(e)$.

2. Differential isomorphisms. In this section, we give some results which correspond to those of [1]-VI-1 and 2 in the case of positive characteristic $p$. In general, these results can be proved similarly to the proofs of the corresponding results of [1], although we have to be careful about separability and need some of the basic results of [2] together with the following three lemmas. Lemma 1 is due to Okugawa.

LEMMA 1. Let $L$ be a differential field with $N \supset L \supset K$. If $N$ and $L$ are finitely generated differential extension fields of $K$ (as differential fields) and $N$ is L-separable, then, for every differential isomorphism $\varphi$ of $L$ over $K, \varphi$ can be extended to a differential isomorphism of $N$ over $K$.

Proof. There exist elements $\eta_{1}, \ldots, \eta_{m}$ of $N$ such that $N=$ $L\left\langle\eta_{1}, \ldots, \eta_{m}\right\rangle$. Let $\mathfrak{p}$ be the defining ideal of $(\eta)=\left(\eta_{1}, \ldots, \eta_{m}\right)$ in the 
differential polynomial ring $L\left\{Y_{1}, \ldots, Y_{m}\right\}$ and $\mathfrak{p}^{\varphi}$ the prime differential ideal in $(\varphi L)\left\{Y_{1}, \ldots, Y_{m}\right\}$ which consists of all the differential polynomials obtained from differential polynomials of $\mathfrak{p}$ by applying $\varphi$ to the coefficients. Since $\mathfrak{p}$ is $L$-separable, $\mathfrak{p}^{\varphi}$ is $(\varphi L)$-separable. Let $\zeta_{1}, \ldots, \zeta_{r}$ be elements of $L$ such that $L=K\left\langle\zeta_{1}, \ldots, \zeta_{r}\right\rangle$. Then, $\varphi L=K\left\langle\varphi \zeta_{1}, \ldots, \varphi \zeta_{r}\right\rangle$ is a finitely generated differential extension field of $K$ in $U$. By Theorem 4 of [2]-IV-4, $U$ is a universal differential extension field of $\varphi L$ and hence, $\mathfrak{p}^{\varphi}$ has a generic zero $\left(\eta^{\prime}\right)=\left(\eta_{1}^{\prime}, \ldots, \eta_{m}^{\prime}\right)$ with $\eta_{k}^{\prime} \in U(1 \leq k \leq m)$. Define the mapping $\Psi$ of $N=L\langle\eta\rangle$ onto $(\varphi L)\left\langle\eta^{\prime}\right\rangle$ as $\Psi(A(\eta) / B(\eta))=A^{\varphi}\left(\eta^{\prime}\right) / B^{\varphi}\left(\eta^{\prime}\right)$, where $A(Y), B(Y) \in$ $L\left\{Y_{1}, \ldots, Y_{m}\right\}$ with $B(\eta) \neq 0$ and, $A^{\varphi}(Y)$ and $B^{\varphi}(Y)$ are differential polynomials obtained by applying $\varphi$ to the coefficients of $A(X)$ and $B(X)$, respectively. This mapping is well defined, and it is straightforward to show that $\Psi$ is a differential isomorphism of $N$ into $U$ extending $\varphi$.

By Corollary 2 to Theorem 6 of [2]-III-6, the following lemma can be proved.

LeMma 2. $N$ and $U_{c}$ are linearly disjoint over $N_{c}$.

LEMMA 3. If $N$ is finitely $K$-separable (that is, finitely generated and separable over $K$ as a field), then the algebraic closure $K^{0}$ of $K$ in $N$ is of finite degree over $K$, and $N$ is finitely $K^{0}$-regular (that is, finitely generated and regular over $K^{0}$ as a field).

Proof. Since $N$ is finitely $K$-separable, $K^{0}$ is separably algebraic over $K$ and a differential subfield of $N$ (see [2]).

Let $\left(x_{j} ; j \in J\right)$ and $\left(x_{j}^{\prime} ; j \in J\right)$ be two families of elements of $U$ with a common set of indices $J$. If there is a differential homomorphism $\varphi$ over $N$ of $N\left\{x_{j} ; j \in J\right\}$ onto $N\left\{x_{j}^{\prime} ; j \in J\right\}$ such that $\varphi\left(x_{j}\right)=x_{j}^{\prime}$ $(j \in J)$, then $\left(x_{j}^{\prime} ; j \in J\right)$ is said to be a differential specialization of $\left(x_{j} ; j \in J\right)$ over $N$.

Let $\left(\sigma_{j} ; j \in J\right)$ and $\left(\sigma_{j}^{\prime} ; j \in J\right)$ be two families of differential isomorphisms of $N$ with a common set of indices $J$. It is easy to see that the following three conditions are equivalent (cf. [1]-VI-1).

(a) $\left(\sigma_{j}^{\prime} x ; j \in J, x \in N\right)$ is a differential specialization over $N$ of $\left(\sigma_{j} x ; j \in J, x \in N\right)$.

(b) $\left(\sigma_{j}^{\prime} x ; j \in J, x \in N\right)$ is a specialization over $N$ of $\left(\sigma_{j} x ; j \in J\right.$, $x \in N)$. 
(c) The differential isomorphisms $\sigma_{j}^{\prime} \sigma_{j}^{-1}$ of $\sigma_{j} N$ onto $\sigma_{j}^{\prime} N(j \in J)$ and $\mathrm{id}_{N}$ are compatible, that is, there is a ring homomorphism $h$ of $N\left[\bigcup_{j \in J} \sigma_{j} N\right]$ onto $N\left[\bigcup_{j \in J} \sigma_{j}^{\prime} N\right]$ such that $h$ is a common extension of $\mathrm{id}_{N}$ and all of $\sigma_{j}^{\prime} \sigma_{j}^{-1}(j \in J)$.

If these conditions are satisfied, then $\left(\sigma_{j}^{\prime} ; j \in J\right)$ is said to be a specialization of $\left(\sigma_{j} ; j \in J\right)$ and, in this case, we use the notation $\left(\sigma_{j} ; j \in J\right) \rightarrow\left(\sigma_{j}^{\prime} ; j \in J\right)$. This specialization is called generic and denoted by $\left(\sigma_{j} ; j \in J\right) \leftrightarrow\left(\sigma_{j}^{\prime} ; j \in J\right)$ if $\left(\sigma_{j} ; j \in J\right)$ is also a specialization of $\left(\sigma_{j}^{\prime} ; j \in J\right)$.

Let $(x)=\left(x_{k} ; k \in J^{\prime}\right)$ be a family of elements of $N$ such that $N=K\langle x\rangle=K\left\langle x_{k} ; k \in J^{\prime}\right\rangle$, where $J^{\prime}$ is a set of indices. Then, $\left(\sigma_{j}\right.$; $j \in J) \rightarrow\left(\sigma_{j}^{\prime} ; j \in J\right)$ if and only if $\left(\sigma_{j}^{\prime} x_{k} ; j \in J, k \in J^{\prime}\right)$ is a differential specialization of $\left(\sigma_{j} x_{k} ; j \in J, k \in J^{\prime}\right)$ over $N$.

A differential isomorphism $\sigma$ of $N$ over $K$ is said to be isolated if every differential isomorphism $\tau$ such that $\tau \rightarrow \sigma$ is a generic specialization of $\sigma$.

In the rest of this section, we assume that $N$ is finitely $K$-separable.

Proposition 1. (a) If $\sigma$ is a differential isomorphism of $N$ over $K$, then $\operatorname{tr} \operatorname{deg} N \cdot \sigma N / N \leq \operatorname{tr} \operatorname{deg} N / K$. The equality holds if and only if $\sigma$ is isolated.

(b) If $\sigma^{\prime}$ is a specialization of a differential isomorphism $\sigma$ of $N$ over $K$, then $\operatorname{tr} \operatorname{deg} N \cdot \sigma^{\prime} N / N \leq \operatorname{tr} \operatorname{deg} N \cdot \sigma N / N$. The equality holds if and only if $\sigma \leftrightarrow \sigma^{\prime}$.

(c) There exist finitely many isolated differential isomorphisms $\sigma_{1}, \ldots, \sigma_{t}$ of $N$ over $K$ such that every differential isomorphism of $N$ over $K$ is a specialization of one and only one of them. If $N$ is $K$-regular, then $t=1$.

Proof. Since $N$ is finitely $K$-separable, there exist elements $z_{1}, \ldots, z_{n}$ of $N$ such that $N=K\left(z_{1}, \ldots, z_{n}\right)$. Let $\mathfrak{p}$ be the defining differential ideal of $\left(z_{1}, \ldots, z_{n}\right)$ in $K\left\{Z_{1}, \ldots, Z_{n}\right\}$. By Theorem 7 of [2]-III-8 and its corollary, we see the following facts; (1) $N \mathfrak{p}$ is a perfect differential ideal of $N\left\{Z_{1}, \ldots, Z_{n}\right\}$ with finitely many $N$-separable components $\mathfrak{P}_{1}, \ldots, \mathfrak{P}_{t}$ ( $t$ is 1 if $N$ is $K$-regular). (2) Every generic zero of $\mathfrak{p}$ is a zero of one and only one of $\mathfrak{P}_{1}, \ldots, \mathfrak{P}_{t}$. (3) For each $k$ $(1 \leq k \leq t)$, every generic zero of $\mathfrak{P}_{k}$ is a generic zero of $\mathfrak{p}$.

(4) $\operatorname{dim} \mathfrak{P}_{k}$, that is, the transcendence degree of a generic zero of $\mathfrak{P}_{k}$ over $N$, is equal to $\operatorname{dim} \mathfrak{p}$. The rest of the proof is similar to that of Proposition 1 of [1]-VI-1. 
Let $L$ and $M$ be two extensions of $K$. We say that $L$ and $M$ are algebraically disjoint over $K$ if algebraically independent elements of $L$ over $K$ are also algebraically independent over $M$.

By Proposition 1(a), the following proposition can be proved.

Proposition 2. A differential isomorphism $\sigma$ of $N$ over $K$ is isolated if and only if $N$ and $\sigma N$ are algebraically disjoint over $K$.

Using Lemma 3, we can demonstrate the following proposition by a proof that follows that of Proposition 2 of [1]-VI-1.

Proposition 3. Let $\sigma$ and $\sigma^{\prime}$ be differential isomorphisms of $N$ over $K$ such that $\sigma$ is isolated and $N \supset \sigma^{\prime} K^{0}$ (whence $\left.\sigma^{\prime} K^{0}=K^{0}\right)$. Then we have:

(a) $N \cap \sigma N=K^{0} \cap \sigma K^{0}$.

(b) $\sigma^{\prime}$ is a specialization of $\sigma$ if and only if $\sigma$ coincides with $\sigma^{\prime}$ on $K^{0}$. When this is the case, $N$ and $\sigma N$ are linearly disjoint over $K^{0}$.

COROLlary. (a) Let $\sigma_{1}, \ldots, \sigma_{t}$ be isolated differential isomorphisms of $N$ over $K$ such that every differential isomorphism of $N$ over $K$ is a specialization of one and only one of them. Then the field of invariants of $\sigma_{1}, \ldots, \sigma_{t}$ is $K$.

(b) Let $\sigma$ be an isolated differential isomorphism of $N$ over $K$. If $\sigma \rightarrow \mathrm{id}_{N}$, then the field of invariants of $\sigma$ is $K^{0}$.

Proof. (a) Let $\alpha \in N$ be an invariant of $\sigma_{1}, \ldots, \sigma_{t}$. Then, $\alpha$ is in $K^{0}$ by Proposition 3(a) and every differential isomorphism of $N$ over $K$ leaves $\alpha$ invariant. Suppose that $\alpha$ is not in $K$. By Proposition 3 of [2]-V-2, there exists a differential isomorphism $\tau$ of $K^{0}$ over $K$ such that $\tau \alpha \neq \alpha$. By Lemma 1, $\tau$ can be extended to a differential isomorphism of $N$ over $K$. This is a contradiction.

(b) This is clear.

A differential isomorphism $\sigma$ of $N$ is said to be strong if $\sigma$ leaves invariant every element of the field $C$ of constants of $N$ and the inclusions $\sigma N \subset N \cdot U_{c}$ and $N \subset \sigma N \cdot U_{c}$ hold. The field of constants of $N \cdot \sigma N$ is represented by $C(\sigma)$. By Proposition 7 of [2]-III-7, it is easy to see that a differential isomorphism $\sigma$ of $N$ over $C$ is strong if and only if $N \cdot C(\sigma)=N \cdot \sigma N=\sigma N \cdot C(\sigma)$. 
Proposition 4. If $\sigma$ is a strong differential isomorphism of $N$ over $K$, then $\operatorname{tr} \operatorname{deg} N \cdot \sigma N / N=\operatorname{tr} \operatorname{deg} C(\sigma) / C$ and $C(\sigma)$ has finite transcendence degree over $C$.

Proof. Since $N$ is finitely generated over $K$ as a field, $\operatorname{tr} \operatorname{deg} N \cdot \sigma N / N$ is finite. Since $N \cdot \sigma N=N \cdot C(\sigma)$, the proposition follows from Lemma 2.

Proposition 5. Each strong differential isomorphism of $N$ can be extended to a unique differential automorphism of $N \cdot U_{c}$ over $U_{c}$. Conversely, the restriction to $N$ of each differential automorphism of $N \cdot U_{c}$ over $U_{c}$ is strong.

Proof. Let $\sigma$ be a differential isomorphism of $N$ over $C$. By Lemma 2, $\sigma N$ and $U_{c}$ are linearly disjoint over $C$. Hence, $\sigma$ can be extended to a unique differential isomorphism $\sigma^{\prime}$ of $N \cdot U_{c}$ onto $\sigma N \cdot U_{c}$ over $U_{c}$. When $\sigma$ is strong, we have $\sigma N \cdot U_{c}=N \cdot U_{c}$ and $\sigma^{\prime}$ is a differential automorphism of $N \cdot U_{c}$. The converse is clear.

By virtue of this proposition, every strong differential isomorphism of $N$ is identified with the unique differential automorphism of $N \cdot U_{c}$ over $U_{c}$. This identification defines a canonical product of strong differential isomorphisms of $N$.

Proposition 6. Let $\sigma$ and $\tau$ be two strong differential isomorphisms of $N$. Then, $C(\sigma) C(\sigma \tau)=C(\sigma) C(\tau)=C(\sigma \tau) C(\tau)$ and $C\left(\sigma^{-1}\right)=$ $C(\sigma)$.

Proof. Using Proposition 7 of [2]-III-7, we can demonstrate this proposition by a proof that follows that of Proposition 5 of [1]-VI-2.

Proposition 7. Every specialization of a strong differential isomorphism of $N$ is strong.

Proof. Using Theorem 6 of [2]-III-6, we can demonstrate this proposition by a proof that follows that of Proposition 6 of [1]-VI-2.

REMARK. It follows from the way of the proof of Proposition 7 that, if $\sigma$ is a differential isomorphism of $N$ over $C$ satisfying $N \subset \sigma N \cdot U_{c}$ (respectively $\sigma N \subset N \cdot U_{c}$ ), then every specialization $\tau$ of $\sigma$ satisfies $N \subset \tau N \cdot U_{c}$ (respectively $\tau N \subset N \cdot U_{\mathcal{C}}$ ). 
For a generic specialization $\sigma^{\prime}$ of a strong differential isomorphism $\sigma$ of $N$, there exists a unique differential isomorphism $h$ of $N \cdot \sigma N$ onto $N \cdot \sigma^{\prime} N$ over $N$ with $h(\sigma x)=\sigma^{\prime} x(x \in N)$. The restriction of $h$ to $C(\sigma)$ is a field isomorphism of $C(\sigma)$ onto $C\left(\sigma^{\prime}\right)$ over $C$. This isomorphism is said to be induced by the generic specialization and denoted by $S_{\sigma^{\prime}, \sigma}$.

The following Proposition 8, Proposition 9 and its corollary can be demonstrated by proofs that follow those of Proposition 7, Proposition 8 and its corollary of [1]-VI-2.

Proposition 8. Let $\sigma$ be a strong differential isomorphism of $N$.

(a) If $\sigma^{\prime}$ is a generic specialization of $\sigma$ and $\sigma^{\prime \prime}$ is a generic specialization of $\sigma^{\prime}$, then $S_{\sigma^{\prime \prime}, \sigma^{\prime}} S_{\sigma^{\prime}, \sigma}=S_{\sigma^{\prime \prime}, \sigma}$.

(b) If $S$ is a field-isomorphism over $C$ of $C(\sigma)$ onto a subfield $C^{\prime}$ of $U_{c}$, then there exists a unique generic specialization $\sigma^{\prime}$ of $\sigma$ such that $C^{\prime}=C\left(\sigma^{\prime}\right)$ and $S=S_{\sigma^{\prime}, \sigma}$.

Proposition 9. Let $\sigma, \sigma^{\prime}, \tau, \tau^{\prime}$ be strong differential isomorphisms of $N$.

(a) If $(\sigma, \tau) \rightarrow\left(\sigma^{\prime}, \tau^{\prime}\right)$, then $\left(\sigma^{-1}, \sigma^{-1} \tau\right) \rightarrow\left(\sigma^{\prime-1}, \sigma^{\prime-1} \tau^{\prime}\right)$.

(b) Let $\sigma \leftrightarrow \sigma^{\prime}$ and $\tau \leftrightarrow \tau^{\prime}$. Then, $(\sigma, \tau) \rightarrow\left(\sigma^{\prime}, \tau^{\prime}\right)$ if and only if the induced isomorphisms $S_{\sigma^{\prime}, \sigma}$ and $S_{\tau^{\prime}, \tau}$ are compatible.

(c) Let $\sigma \leftrightarrow \sigma^{\prime}$ and $\tau \leftrightarrow \tau^{\prime}$. If a homomorphism $h$ of a subring of $U_{c}$ into another and the induced isomorphisms $S_{\sigma^{\prime}, \sigma}, S_{\tau^{\prime}, \tau}$ are compatible, then $\sigma^{-1} \leftrightarrow \sigma^{-1}$ and $\sigma^{-1} \tau \rightarrow \sigma^{-1} \tau^{\prime}$; when the latter specialization is generic, $h$ and the induced isomorphisms $S_{\sigma^{\prime-1}, \sigma^{-1}}, S_{\sigma^{\prime-1} \tau^{\prime}, \sigma^{-1} \tau}$ aré compatible.

CoRollaRy. Let $\sigma, \sigma^{\prime}, \tau, \tau^{\prime}$ be differential isomorphisms of $N$ and let $\sigma, \tau$ be strong.

(a) If $\sigma \rightarrow \sigma^{\prime}$, then $\sigma^{-1} \rightarrow \sigma^{\prime-1}$. When the former specialization is generic, then so is the latter and $S_{\sigma^{\prime}, \sigma}=S_{\sigma^{\prime-1}, \sigma^{-1}}$.

(b) If $\sigma \leftrightarrow \sigma^{\prime}, \tau \leftrightarrow \tau^{\prime}$ and if the induced isomorphisms $S_{\sigma^{\prime}, \sigma}, S_{\tau^{\prime}, \tau}$ are compatible, then $\sigma \tau \rightarrow \sigma^{\prime} \tau^{\prime}$. When $\sigma \tau \leftrightarrow \sigma^{\prime} \tau^{\prime}$ and $h$ is a homomorphism of a subring of $U_{c}$ into another such that $h, S_{\sigma^{\prime}, \sigma}$ and $S_{\tau^{\prime}, \tau}$ are compatible, $h$ and $S_{\sigma^{\prime} \tau^{\prime}, \sigma \tau}$ are compatible.

3. Strongly normal differential extensions and Galois groups. We denote the set of all differential isomorphisms of $N$ over $K$ by $G(N / K)$. For $\sigma \in G(N / K)$, the field of constants of $N \cdot \sigma N$ is represented by $C(\sigma)$. 
We say that $N$ is strongly normal over $K$ if $N$ is finitely $K$-separable and every differential isomorphism of $N$ over $K$ is strong. If $N$ is strongly normal over $K$, then the field of constants $C$ of $N$ coincides with the field of constants $K_{c}$ of $K$ by Corollary (a) to Proposition 3. Moreover, by Proposition 5, we see that $G(N / K)$ has a canonical group structure.

It is easy to see that Picard-Vessiot extensions are strongly normal extensions. No examples of strongly normal extensions which are not Picard-Vessiot extensions are so far known however.

In the rest of this section, we assume that $N$ is finitely $K$-separable.

Proposition 10. Let $\sigma_{1}, \ldots, \sigma_{t}$ be isolated differential isomorphisms of $N$ over $K$ such that every element of $G(N / K)$ is a specialization of one of $\sigma_{1}, \ldots, \sigma_{t}$. If $C=K_{c}$ and $\sigma_{k} N \subset N \cdot U_{c}(1 \leq k \leq t)$, then $N$ is strongly normal over $K$.

Proof. Let $\sigma$ be one of $\sigma_{1}, \ldots, \sigma_{t}$. By Proposition 2, $N$ and $\sigma N$ are algebraically disjoint over $K$ and hence, $N \cdot \sigma N$ is finitely separable over $\sigma N$. Therefore, by Lemma 1, the differential isomorphism $\sigma^{-1}$ of $\sigma N$ onto $N$ can be extended to a differential isomorphism $h$ of $N \cdot \sigma N$ into $U$. Let $\tau$ be the restriction of $h$ to $N$. Then, it is a differential isomorphism of $N$. We see that $h$ is a differential isomorphism of $N \cdot \sigma N$ onto $\tau N \cdot N$ over $K$ with $h N=\tau N, h(\sigma N)=N$ and $h(C(\sigma))=$ $C(\tau)$. Since $\tau$ is a specialization of some $\sigma_{k}$ and $\sigma_{k} N \subset N \cdot U_{c}$, we have the inclusion $\tau N \subset N \cdot C(\tau)$ by Remark to Proposition 7. Thus, $N=$ $h^{-1}(\tau N) \subset h^{-1}(N \cdot C(\tau))=\sigma N \cdot C(\sigma)$. Therefore, $\sigma_{1}, \ldots, \sigma_{t}$ are strong and, by Proposition 7, we see that every differential isomorphism of $N$ over $K$ is strong.

Proposition 11. If $\sigma$ is a strong differential isomorphism of $N$ over $K$, then $C(\sigma)$ is finitely generated over $C$ as a field. Moreover, if $\sigma$ is isolated, then $C(\sigma)$ is separable over $C$.

Proof. By Proposition 4, $C(\sigma)$ has finite transcendence degree over $C$. Let $\gamma_{1}, \ldots, \gamma_{s}$ be a transcendence basis of $C(\sigma)$ over $C$. Since $N$ and $C(\sigma)$ are linearly disjoint over $C, \gamma_{1}, \ldots, \gamma_{s}$ are algebraically independent over $N$. As $N \cdot \sigma N=N \cdot C(\sigma)$ is finitely generated over $N, N \cdot C(\sigma)$ is algebraic of finite degree, say $r$, over $N\left(\gamma_{1}, \ldots, \gamma_{s}\right)$. Every element of $C(\sigma)$ is algebraic of degree not greater than $r$ over $N\left(\gamma_{1}, \ldots, \gamma_{s}\right)$ and hence, over $C\left(\gamma_{1}, \ldots, \gamma_{s}\right)$ by Proposition 6 of [2]III-7. Therefore, $C(\sigma)$ is algebraic of finite degree over $C\left(\gamma_{1}, \ldots, \gamma_{s}\right)$. Thus, $C(\sigma)$ is finitely generated over $C$. 
Assume moreover that $\sigma$ is isolated. Then, by Proposition 2, $N$ and $\sigma N$ are algebraically disjoint over $K$. Since $\sigma N$ is finitely $K$-separable, $N \cdot \sigma N$ is finitely $N$-separable (see Theorem 8 and Proposition 26 of [6]-I). $N$ and $C(\sigma)$ are linearly disjoint over $C$. Since the order of inseparability $i(C(\sigma) / C)$ of $C(\sigma)$ over $C$ (see [6]-I-8) coincides with $i(N \cdot \sigma N / N)=1, C(\sigma)$ is separable over $C$.

In the case where $N$ is strongly normal over $K$, we have already described the group structure of $G(N / K)$. For the sake of simplicity, this group will be denoted by $G$.

THEOREM 1. If $N$ is strongly normal over $K$, then $G$ has a pre- $C$-set structure (see [1]-V-2). This pre-C-set structure and the group structure of $G$ define a $C$-group (i.e. algebraic group defined over $C$ ) structure on $G$ relative to the universal field $U_{c}$ (see [1]-V-3). The dimension of the $C$-group $G$ equals the transcendence degree of $N$ over $K$.

Proof. We must verify the axioms of [1]-V-2 and 3.

For any $\sigma \in G, C(\sigma)$ is finitely generated over $C$ by Proposition 11. The relation $\sigma \rightarrow \sigma^{\prime}$ (for $\sigma, \sigma^{\prime} \in G$ ) is reflexive and transitive by definition. For each pair $\sigma, \sigma^{\prime} \in G$ with $\sigma \leftrightarrow \sigma^{\prime}$, associate the field-isomorphism $S_{\sigma^{\prime}, \sigma}$ over $C$. We can see that these data satisfy the axioms AS1 and 2 by virtue of Proposition 1(b), (c) and Proposition 8. Thus, $C$ has a pre- $C$-set structure.

By Proposition 6, Proposition 9 and its corollary (a), (b), we can verify the axioms AG1 and AG2(a), (c). In order to verify the axiom AG3, let $\sigma \in G$ be isolated with $\sigma \rightarrow \mathrm{id}_{N}$; in particular, $\sigma$ is generic for the identity component of $G$ over $C$. Since $N$ is regular over $K^{0}$, so is $\sigma N$ over $\sigma K^{0}$. By Proposition 3, we see that $\sigma K^{0}=K^{0}$ and that $N$ and $\sigma N$ are linearly disjoint over $K^{0}$. Therefore, $N \cdot \sigma N=N \cdot C(\sigma)$ is $N$-regular (see Theorem 5 of [6]-I-7). Since $N$ and $C(\sigma)$ are linearly disjoint over $C$, we conclude that $C(\sigma)$ is $C$-regular by Proposition 11 . Thus, AG3 is satisfied.

Now, we shall verify the axioms AG2(b) and (d). Let $\sigma, \sigma^{\prime}, \tau, \tau^{\prime} \in G$ with $\sigma \rightarrow \sigma^{\prime}$ and $\tau \rightarrow \tau^{\prime}$. Since $N$ is finitely $K$-separable, there exist elements $z_{1}, \ldots, z_{n}$ of $N$ such that $N=K\left\langle z_{1}, \ldots, z_{n}\right\rangle=K\left(z_{1}, \ldots, z_{n}\right)$. Denote by $\mathfrak{p}$ and $\mathfrak{q}$ the defining ideals over $N$ of $\left(\sigma^{-1} z_{1}, \ldots, \sigma^{-1} z_{n}\right)$ and $\left(\tau z_{1}, \ldots, \tau z_{n}\right)$ in the differential polynomial rings $N\left\{X_{1}, \ldots, X_{n}\right\}$ and $N\left\{Y_{1}, \ldots, Y_{n}\right\}$ respectively. Then, by Theorem 7 of [2]-III-8 and Theorem 3 of [5], $N_{\Delta} \mathfrak{p}$ and $N_{\Delta} \mathfrak{q}$ have finitely $N_{\Delta}$-regular compo- 
nents $\mathfrak{p}_{1}, \ldots, \mathfrak{p}_{r}$ and $\mathfrak{q}_{1}, \ldots, \mathfrak{q}_{s}$ respectively. Each differential ideal $\mathfrak{r}_{k t}=$ $\left(\mathfrak{p}_{k}, \mathfrak{q}_{t}\right)(1 \leq k \leq r, 1 \leq t \leq s)$ of $N_{\Delta}\left\{X_{1}, \ldots, X_{n}, Y_{1}, \ldots, Y_{n}\right\}$ is prime and $N_{\Delta}$-regular (see Proposition 1 of [2]-IV-1), and it has a generic zero $\left(x_{1}^{(k, t)}, \ldots, x_{n}^{(k, t)}, y_{1}^{(k, t)}, \ldots, y_{n}^{(k, t)}\right)$ with $x_{j}^{(k, t)}, y_{j}^{(k, t)} \in U(1 \leq j \leq n)$. Since $\left(x_{1}^{(k, t)}, \ldots, x_{n}^{(k, t)}\right)$ is a generic zero of $\mathfrak{r}_{k t} \cap N_{\Delta}\left\{X_{1}, \ldots, X_{n}\right\}=\mathfrak{p}_{k}$, whence of $\mathfrak{p}_{k} \cap N\left\{X_{1}, \ldots, X_{n}\right\}=\mathfrak{p},\left(x_{1}^{(k, t)}, \ldots, x_{n}^{(k, t)}\right)$ is a generic specialization of $\left(\sigma^{-1} z_{1}, \ldots, \sigma^{-1} z_{n}\right)$ over $N$ and over $K$. Therefore, there exists a strong differential isomorphism $\sigma_{k t}^{-1}$ of $N$ over $K$ with $\sigma_{k t}^{-1} z_{j}=$ $x_{j}^{(k, t)}(1 \leq j \leq n)$. Then, $\sigma^{-1} \leftrightarrow \sigma_{k t}^{-1}$ by Lemma 2 . Similarly, there exists a strong differential isomorphism $\tau_{k t}$ of $N$ over $K$ such that $\tau_{k t} z_{j}=$ $y_{j}^{(k, t)}(1 \leq j \leq n)$ and $\tau \leftrightarrow \tau_{k t}$. By our assumption $\sigma \rightarrow \sigma^{\prime}$ and Proposition 9 , we see that $\sigma^{-1} \rightarrow \sigma^{-1}$ and $\left(\sigma^{\prime-1} z_{1}, \ldots, \sigma^{\prime-1} z_{n}\right)$ is a zero of $\mathfrak{p}$ and hence, of some $\mathfrak{p}_{k}$. Similarly, $\left(\tau^{\prime} z_{1}, \ldots, \tau^{\prime} z_{n}\right)$ is a zero of some $\mathfrak{q}_{t}$. Then, $\left(\sigma^{\prime-1} z_{1}, \ldots, \sigma^{\prime-1} z_{n}, \tau^{\prime} z_{1}, \ldots, \tau^{\prime} z_{n}\right)$ is a zero of some $\mathfrak{r}_{k t}$ and it is a differential specialization of $\left(\sigma_{k t}^{-1} z_{1}, \ldots, \sigma_{k t}^{-1} z_{n}, \tau_{k t} z_{1}, \ldots, \tau_{k t} z_{n}\right)$ over $N_{\Delta}$ and hence, over $N$. Therefore, we have $\left(\tau_{k t}, \sigma_{k t}^{-1}\right) \rightarrow\left(\tau^{\prime}, \sigma^{\prime-1}\right)$ and, by Proposition 9, we see that $\left(\tau_{k t}^{-1}, \tau_{k t}^{-1} \sigma_{k t}^{-1}\right) \rightarrow\left(\tau^{\prime-1}, \tau^{\prime-1} \sigma^{\prime-1}\right)$. If $\sigma_{k t} \tau_{k t} \leftrightarrow \sigma^{\prime} \tau^{\prime}$ and $\tau_{k t} \rightarrow \tau^{\prime}$, then the induced isomorphisms $C\left(\sigma_{k t} \tau_{k t}\right) \rightarrow$ $C\left(\sigma^{\prime} \tau^{\prime}\right)$ and $C\left(\tau_{k t}\right) \rightarrow C\left(\tau^{\prime}\right)$ are compatible. This shows that the axioms AG2(b) and (d) are satisfied, since $\sigma^{-1} \rightarrow \sigma^{\prime-1}$ whenever $\sigma \rightarrow \sigma^{\prime}$.

Finally, let $\sigma$ be an isolated differential isomorphism of $N$ over $K$ with $\sigma \rightarrow \operatorname{id}_{N}$. Since $N$ and $C(\sigma)$ are linearly disjoint over $C$, and $N$ and $\sigma N$ are algebraically disjoint over $K$,

$$
\begin{aligned}
\operatorname{tr} \operatorname{deg} C(\sigma) / C & =\operatorname{trdeg} N \cdot C(\sigma) / N=\operatorname{trdeg} N \cdot \sigma N / N \\
& =\operatorname{trdeg} N / K .
\end{aligned}
$$

The $C$-group $G$ stated in Theorem 1 is called the Galois group of $N$ over $K$.

THEOREM 2. Let $N$ be strongly normal over $K$ and let $C^{\prime}$ be an extension of $C$ in $U_{c}$ such that $U$ is universal over $\left(N C^{\prime}\right)_{\Delta}$. Then, $N C^{\prime}$ is a strongly normal differential extension of $K C^{\prime}$ with the field of constants $C^{\prime}$, and the Galois group $G\left(N C^{\prime} / K C^{\prime}\right)$ is the induced (see [1]$\mathrm{V}$-5) $C^{\prime}$-group of the $C$-group $G$, both these groups being identified with each other by means of their canonical identifications with the group of automorphisms of $N U_{c}$ over $K U_{c}$.

Proof. By [2]-III-6 and 7, it is easy to see that $N C^{\prime}$ is finitely $K C^{\prime}-$ separable and that $\left(K C^{\prime}\right)_{c}=C^{\prime}$. 
In order to prove that $N C^{\prime}$ is strongly normal over $K C^{\prime}$, let $\sigma$ be a differential isomorphism of $N C^{\prime}$ over $K C^{\prime}$. Since the restriction of $\sigma$ to $N$ is strong,

$$
\sigma\left(N C^{\prime}\right)=\sigma N \cdot \sigma C^{\prime} \subset N \cdot U_{c} \cdot C^{\prime}=N C^{\prime} \cdot U_{c} .
$$

Similarly, $N C^{\prime} \subset \sigma\left(N C^{\prime}\right) \cdot U_{c}$. Thus, $N C^{\prime}$ is strongly normal over $K C^{\prime}$. By Theorem 1, $G\left(N C^{\prime} / K C^{\prime}\right)$ is a $C^{\prime}$-group.

It suffices then to prove that this $C^{\prime}$-group $G\left(N C^{\prime} / K C^{\prime}\right)$ is the induced $C^{\prime}$-group of the $C$-group $G(N / K)$. If we denote by $C^{\prime}(\sigma)$ the field of constants of $N C^{\prime} \cdot \sigma\left(N C^{\prime}\right)$, then

$N \cdot C^{\prime}(\sigma)=N C^{\prime} \cdot C^{\prime}(\sigma)=N C^{\prime} \cdot \sigma\left(N C^{\prime}\right)=N \cdot \sigma N \cdot \sigma C^{\prime}=N \cdot C(\sigma) \cdot C^{\prime}$, and $C^{\prime}(\sigma)=C(\sigma) \cdot C^{\prime}$ by Proposition 7 of [2]-III-7. The remaining part of the theorem can be proved similarly to Theorem 2 of [1]-VI-3.

\section{Fundamental theorems.}

Proposition 12. Let $M$ be a differential subfield of $N$ and let $L$ be a differential extension field of $M$. If $L$ is purely inseparably algebraic over $M$, then every element of $G(N / M)$ can be extended uniquely to an element of $G(N L / L)$.

Proof. Since $N L \subset N_{\Delta}$, we see that $U$ is universal over $(N L)_{\Delta}$ by Corollary to Lemma 1 of [2]-IV-2. Clearly $G(L / M)=\left\{\mathrm{id}_{L}\right\}$, and this implies that $G(N L / M)=G(N L / L)$. By Theorem 2 of [5], each differential isomorphism in $G(N / M)$ can be extended to some differential isomorphism in $G(N L / M)$, and this extension is unique since $N L$ is purely inseparable over $N$. Thus, the proposition is established.

The purely inseparably algebraic closure of a differential field $M$ in $M_{\Delta}$ is denoted by $M_{\infty}$ (see $\S 2$ of [5]). The field $M_{\infty}$ is a differential subfield of $M_{\Delta}$. The field of constants of $M_{\infty}$ coincides with the purely inseparably algebraic closure $C_{i}$ of $C$ in $C_{a}$. Since the field of constants of $M$ is $C$, we have $\left(M_{\Delta}\right)_{c}=C_{a}$ by Theorem 1 of [5] and $\left(M_{\infty}\right)_{c} \supset C_{i}$. Since $M_{\infty}$ is purely inseparably algebraic over $M$, so is $\left(M_{\infty}\right)_{c}$ over $C$. Therefore, we have $\left(M_{\infty}\right)_{c}=C_{i}$.

Proposition 13. Let $M$ be a differential field with $N \supset M \supset K$. If $N$ is strongly normal over $K$, then $N M_{\infty}$ is strongly normal over $M_{\infty}$.

Proof. By Theorem 4 of [5], $N M_{\infty}$ is finitely $M_{\infty}$-separable. If $c$ is a constant in $N M_{\infty}$, then since $N M_{\infty}$ is purely inseparable over $N$, 
there is a positive integer $e$ such that $c^{p(e)} \in N$. Thus, $c^{p(e)} \in N_{c}=C$, whence $c \in C_{i}$. Hence, we have $\left(N M_{\infty}\right)_{c}=C_{i}=\left(M_{\infty}\right)_{c}$. Now let $\sigma \in G\left(N M_{\infty} / M_{\infty}\right)$. The restriction $\left.\sigma\right|_{N}$ of $\sigma$ to $N$ is in $G(N / M) \subset$ $G(N / K)$. Therefore, $\sigma\left(N M_{\infty}\right)=\sigma N \cdot M_{\infty} \subset N U_{c} \cdot M_{\infty}=\left(N M_{\infty}\right) \cdot U_{c}$, and $N M_{\infty} \subset \sigma N \cdot U_{c} \cdot M_{\infty}=\sigma\left(N M_{\infty}\right) \cdot U_{c}$. Since $\left(N M_{\infty}\right)_{c}=\left(M_{\infty}\right)_{c}$, it follows that $N M_{\infty}$ is strongly normal over $M_{\infty}$.

Now, $G(N / M)$ is a subset of $G$, which is a $C$-group. The group structure on $G$ is induced by that of $\operatorname{Aut}\left(N U_{c} / K U_{c}\right)$. The $C$-set structure was described above.

We now show that even though $N$ is not necessarily strongly normal over the intermediate differential field $M$, nonetheless, $G(N / M)$ is a $C$-subgroup of $G$. It is easy to see that the field of constants of $K_{\infty}$ is also $C_{i}$. Thus, $G\left(N M_{\infty} / M_{\infty}\right)$ and $G\left(N K_{\infty} / K_{\infty}\right)$ are $C_{i}$-groups. Moreover, the restriction homomorphism is an embedding of the $C_{i}$-group $G\left(N M_{\infty} / M_{\infty}\right)$ into the $C_{i}$-group $G\left(N K_{\infty} / K_{\infty}\right)$. This enables us, indeed, to identify $G\left(N M_{\infty} / M_{\infty}\right)$ with a $C_{i}$-subgroup of $G\left(N K_{\infty} / K_{\infty}\right)$. The restriction homomorphism of $G\left(N K_{\infty} / K_{\infty}\right)$ onto $G(N / K)$ is a $C_{i^{-}}$ isomorphism, mapping $G\left(N M_{\infty} / M_{\infty}\right)$ onto $G(N / M)$. Thus, the latter is a $C_{i}$-subgroup of $G$. It follows that $G(N / M)$ is a $C$-subgroup of $G$ ([1]-V-6). So we have proved the following proposition.

Proposition 14. Let $N$ be strongly normal over $K$ and let $M$ be a differential field with $N \supset M \supset K$. Then $G(N / M)$ is a C-subgroup of $G$.

If $N$ is strongly normal over $K$, then we see by Theorem 2 that the $C$-group $G$ can be identified with the $C_{s}$-group $G\left(N \cdot C_{S} / K \cdot C_{S}\right)$.

Proposition 15. Let $N$ be strongly normal over $K$, and $H$ a $C$ subgroup of $G$. Let $L$ and $L^{\prime}$ be the fields of invariants of $H$ in $N$ and in $N C_{s}$ respectively. Then:

(a) $L^{\prime}$ and $N$ are linearly disjoint over $L$.

(b) $L^{\prime}=L C_{s}$.

Proof. (a) Let $y_{1}, \ldots, y_{s+1}$ be elements of $L^{\prime}$ which are linearly dependent over $N$ and any $s$ elements of which are linearly independent over $N$. Then, there exist nonzero elements $x_{1}, \ldots, x_{s} \in N$ such that $y_{s+1}=\sum_{j=1}^{s} x_{j} y_{j}$. We prove that $x_{1}, \ldots, x_{s}$ are in $L$.

We set $H^{\prime}=\left\{\sigma \in H ; \sigma\right.$ is rational over $\left.C_{s}\right\}$. Suppose that there exists an element $\sigma \in H^{\prime}$ and $r(1 \leq r \leq s)$ such that $\sigma x_{r} \neq x_{r}$; say 
$\sigma x_{1} \neq x_{1}$. Since

$$
y_{s+1}=\sigma y_{s+1}=\sum_{j=1}^{s} \sigma x_{j} \cdot y_{j}
$$

we have

$$
0=\sum_{j=1}^{s}\left(\sigma x_{j}-x_{j}\right) y_{j}
$$

and

$$
0=\sum_{j=1}^{s} u_{j} y_{j}
$$

where $u_{j}=\left(\sigma x_{j}-x_{j}\right) /\left(\sigma x_{1}-x_{1}\right) \in N \cdot \sigma N=N \cdot C(\sigma)(1 \leq j \leq s)$. As $N \cdot C(\sigma)$ is finitely $N$-separable, there exists an element $v$ of $N \cdot C(\sigma)$ such that $\operatorname{Tr}_{N \cdot C(\sigma) / N}(v) \neq 0$. Multiplying (1) by $v$, we obtain

$$
0=\sum_{j=1}^{s} v u_{j} y_{j}
$$

Let $\sigma_{1}, \ldots, \sigma_{t}$ be all the $C$-conjugates of $\sigma$. Then

$$
0=\sum_{j=1}^{s} \sigma_{k}\left(v u_{j}\right) \cdot y_{j} \quad(1 \leq k \leq t)
$$

and

$$
0=\sum_{j=1}^{s}\left(\sum_{k=1}^{t} \sigma_{k}\left(v u_{j}\right)\right) \cdot y_{j}=\sum_{j=1}^{s} \operatorname{Tr}_{N \cdot C(\sigma) / N}\left(v u_{j}\right) y_{j}
$$

Since

$$
\operatorname{Tr}_{N \cdot C(\sigma) / N}\left(v u_{j}\right) \in N \quad(1 \leq j \leq s)
$$

and

$$
\operatorname{Tr}_{N \cdot C(\sigma) / N}\left(v u_{1}\right)=\operatorname{Tr}_{N \cdot C(\sigma) / N}(v) \neq 0,
$$

we see that $y_{1}, \ldots, y_{s}$ are linearly dependent over $N$, which yields a contradiction.

Thus, we conclude that $\sigma x_{j}=x_{j}(1 \leq j \leq s)$ for every $\sigma \in H^{\prime}$. Set $H^{\prime \prime}=\left\{\tau \in G ; \tau x_{j}=x_{j}(1 \leq j \leq s)\right\}$. Then, $H^{\prime} \subset H^{\prime \prime}$ and, by Proposition 14, we see that $H^{\prime \prime}$ is a $C$-closed subgroup of $G$ (see [1]$\mathrm{V}$ ). Since $H^{\prime}$ is dense in $H$ (see Corollary to Proposition 3 of [1]-V-7), $H \subset H^{\prime \prime}$ and $x_{j} \in L(1 \leq j \leq s)$.

(b) Let $\left\{c_{j} ; j \in J\right\}$ be a linear basis of $C_{s}$ over $C$. Then, each $y \in L^{\prime}$ is written in the form $y=\sum_{j \in J^{\prime}} n_{j} c_{j}$ with $n_{j} \in N$ and a finite subset $J^{\prime}$ of $J$. Thus, $y$ and $\left\{c_{j} ; j \in J^{\prime}\right\}$ are linearly dependent over $N$. By 
part (a), they are linearly dependent over $L$ and hence, there exist $w$, $w_{j}\left(j \in J^{\prime}\right)$ in $L$ such that $w y=\sum_{j \in J^{\prime}} w_{j} c_{j}$. Since $\left\{c_{j} ; j \in J\right\}$ is linearly independent over $C, w$ cannot be zero and

$$
y=\sum_{j \in J^{\prime}}\left(w_{j} / w\right) c_{j}
$$

This implies that $L^{\prime} \subset L C_{s}$ and hence, $L^{\prime}=L C_{s}$.

THEOREM 3. Let $N$ be strongly normal over $K$. Then:

(a) If $M$ is a differential field with $N \supset M \supset K$, then $G(N / M)$ is a $C$-closed subgroup of $G$. Moreover, the field $L$ of invariants in $N$ of $G(N / M)$ is the purely inseparably algebraic closure in $N$ of $M$ and $G(N / M)=G(N / L)$.

(b) If $H$ is a $C$-closed subgroup of $G$, then the field $L$ of invariants in $N$ of $H$ is purely inseparably algebraically closed in $N$ and $H=$ $G(N / L)$.

Proof. (a) By Propositions 12 and $13, N M_{\infty}$ is strongly normal over $M_{\infty}$ and $G\left(N M_{\infty} / M_{\infty}\right)$ is $C$-isomorphic to the $C$-closed subgroup $G(N / M)$ of $G$. By Corollary (a) of Proposition 3, $M_{\infty}$ is the field of invariants in $N M_{\infty}$ of $G\left(N M_{\infty} / M_{\infty}\right)$; hence every element of $L$ is purely inseparably algebraic over $M$. Since $G(N / M)$ leaves invariant every element of $M$ and $L$ is purely inseparably algebraic over $M$, we see that $L$ is the purely inseparably algebraic closure in $N$ of $M$ and $G(N / M)=G(N / L)$.

(b) Applying part (a) to the field $L$ of invariants in $N$ of $H$ instead of $M$, we see that $G(N / L)$ is a $C$-closed subgroup of $G$ and $G(N / L) \supset H$.

We now prove that $H=G(N / L)$.

We can see that $H$ can be regarded as a $C_{a}$-subset (see [1]-V-3, p. 227) of the $C_{a}$-group $G\left(N C_{a} / K C_{a}\right)=G(N / K)$, by the assumption and Theorem 2. Let $L^{\prime}$ be the set of invariants in $N C_{a}$ of $H$. Since $C_{a}=\left(C_{a}\right)_{s}$, the equality $H=G\left(N C_{a} / L^{\prime}\right)$ can be established by the same argument used in the proof of Theorem 3 of [1]-VI-4.

We prove that $N C_{a}$ is separable over $L^{\prime}$. Let $y_{1}, \ldots, y_{s+1}$ be elements of $L^{\prime}$ which are linearly dependent over $\left(N C_{a}\right)^{p}$ and any $s$ of which are linearly independent over $\left(N C_{a}\right)^{p}$. Then, there exist $s$ elements $\alpha_{1}, \ldots, \alpha_{s}$ of $N C_{a}$ such that $y_{s+1}=\sum_{k=1}^{s} \alpha_{k}^{p} y_{k}$. Set $H^{\prime \prime}=$ $\left\{\sigma \in H ; \sigma\right.$ is rational over $\left.C_{a}\right\}$. Each element $\tau$ of $H^{\prime \prime}$ is a differential automorphism of $N C_{a}$ over $K C_{a}$; hence we see that

$$
y_{s+1}=\sum_{k=1}^{s}\left(\tau \alpha_{k}\right)^{p} y_{k}, \quad \sum_{k=1}^{s}\left(\tau \alpha_{k}-\alpha_{k}\right)^{p} y_{k}=0
$$


and $\tau \alpha_{k}=\alpha_{k}(1 \leq k \leq s)$. Since the set $\left\{\sigma \in G\left(N C_{a} / K C_{a}\right) ; \sigma \alpha_{k}=\alpha_{k}\right.$ $(1 \leq k \leq s)\}$ is $C_{a}$-closed and $H^{\prime \prime}$ is dense in $H$ (see Corollary to Proposition 3 of [1]-V-7), we have $\sigma \alpha_{k}=\alpha_{k}$ for every $\sigma \in H$ and hence, $\alpha_{k} \in L^{\prime}(1 \leq k \leq s)$.

Let $H$ be regarded as a $C_{i}$-subgroup (see [1]-V-3) of the $C_{i}$-group $G\left(N C_{i} / K C_{i}\right)=G(N / K)$ and let $L^{*}$ be the set of invariants in $N C_{i}$ of $H$. As $\left(C_{i}\right)_{s}=C_{a}$, Proposition 15 implies that $L^{\prime}$ and $N C_{i}$ are linearly disjoint over $L^{*}$ and $L^{\prime}=L^{*} C_{a}$. Since $N C_{a}$ is finitely $L^{\prime}$-separable over $L^{\prime}$, we see that $N C_{i}$ is finitely $L^{*}$-separable and hence, strongly normal over $L^{*}$. Therefore, by Theorem 2 , we have

$$
G\left(N C_{i} / L^{*}\right)=G\left(\left(N C_{i}\right) C_{a} / L^{*} C_{a}\right)=G\left(N C_{a} / L^{\prime}\right)=H .
$$

By Proposition $13, N L_{\infty}$ is strongly normal over $L_{\infty}$ and $G\left(N L_{\infty} / L_{\infty}\right)$ $=G(N / L)$ is a $C$-closed subgroup of $G(N / K)$. When we regard $H$ as a $C_{i}$-subgroup of $G\left(N L_{\infty} / L_{\infty}\right)$, the field $M$ of invariants in $N L_{\infty}$ of $H$ contains $L_{\infty}$. Since $N L_{\infty}$ is purely inseparably algebraic over $N$, for each $x \in M$, there is an integer $e$ with $x^{p(e)} \in N$. Thus, we have $\sigma\left(x^{p(e)}\right)=(\sigma x)^{p(e)}=x^{p(e)}$ for every $\sigma \in H$ and we have $x^{p(e)} \in L$ i.e. $x \in L_{\infty}$. Therefore, we conclude that $M=L_{\infty}, L_{\infty} \supset L^{*}$ and $L^{*}$ is purely inseparably algebraic over $L C_{i}$. Then, by Proposition 12, part (a) of the present theorem and (2), we have

$$
G(N / L)=G\left(N C_{i} / L C_{i}\right)=G\left(N C_{i} / L^{*}\right)=H .
$$

By the proof of part (b) of Theorem 3, the following corollary is obvious.

COROLlaRY. Let $N$ be strongly normal over $K$. If the field of constants of the ground field $K$ is perfect, then:

(a) If $L$ is a differential field with $N \supset L \supset K$ and $L$ is purely inseparably algebraically closed in $N$, then $N$ is strongly normal over $L, G(N / L)$ is a C-subgroup of $G($ see $[1]-V-3)$ and the field of invariants in $N$ of $G(N / L)$ is $L$.

(b) If $H$ is a $C$-subgroup of $G$ and $L$ is the field of invariants in $N$ of $H$, then $L$ is purely inseparably algebraically closed in $N, N$ is strongly normal over $L$ and $H=G(N / L)$.

REMARK. In order that Theorem 3 has the usual formulation of the fundamental theorem of Galois theory, some extra condition is necessary (see Example 2 of $\S 5$ below). The condition $C=C_{i}$ is such an example. 
THEOREM 4. Let $N$ be strongly normal over $K$. If $L$ is a differential field with $N \supset L \supset K$ and $N$ is separable over $L$, then the following four conditions are equivalent.

(a) $L$ is strongly normal over $K$.

(b) For every $\alpha \in L-K$, there exists a strong differential isomorphism $\tau$ of $L$ over $K$ such that $\tau \alpha \neq \alpha$.

(c) $G(N / L)$ is a normal subgroup of $G$.

(d) For every $\sigma \in G$, the inclusion $\sigma L \subset L U_{c}$ holds.

When these conditions are satisfied, the sequence

$$
G(N / L) \stackrel{l}{\rightarrow} G \stackrel{\varphi}{\rightarrow} G(L / K) \rightarrow\{\mathrm{id}\}
$$

is exact where 1 denotes the canonical embedding and $\varphi$ is a homomorphism defined by $\varphi(\sigma)=\left.\sigma\right|_{L}(\sigma \in G)$. Moreover, $\varphi$ becomes a C-homomorphism.

Proof. First, assume that (a) is satisfied. Then, the field of invariants of $G(L / K)$ in $L$ is $K$ (see Theorem 3) and (b) is satisfied.

Next, assume that (b) is satisfied. If we denote by $Z$ the normalizer of $G(N / L)$ in $G(N / K)$, then $Z$ is a $C$-closed subgroup of $G(N / K)$ containing $G(N / L)$ (see [1]-V-10). Let $M$ be the field of invariants in $N$ of $Z$. Suppose that $M \neq K$. We can take an element $\alpha \in M-K \subset L-K$. By (b), there exists a strong differential isomorphism $\tau$ of $L$ over $K$ with $\tau \alpha \neq \alpha$. By Proposition 5, we see that $\tau$ can be extended to a differential isomorphism $\tau^{\prime}$ of $L C_{i}$ over $K C_{i}$. Since $N C_{i}$ is finitely $L C_{i}$-separable (see Theorem 2), it follows from Lemma 1 that $\tau^{\prime}$ can be extended to a differential isomorphism $\sigma$ of $N C_{i}$ over $K C_{i}$. For every $\rho \in G(N / L)$ and every $\beta \in L$, we have $\sigma \beta=\tau \beta \in L U_{c}, \rho \sigma \beta=\sigma \beta$ and $\sigma^{-1} \rho \sigma \beta=\beta$. Therefore, $\sigma^{-1} \rho \sigma \in G(N / L)$ and $\sigma \in Z=G(N / M)$ (see Theorem 3). This contradicts the inequality $\sigma \alpha \neq \alpha$, and we conclude that $M=K$. Then, $Z=G$ and $G(N / L)$ is a normal subgroup of $G$. Thus, (c) is satisfied.

Now, assume that (c) is satisfied. Let $\sigma \in G$ and let $\beta \in L$. Since $\sigma^{-1} \rho \sigma \in G(N / L)$ for every $\rho \in G(N / L)$, we have $\sigma^{-1} \rho \sigma \beta=\beta$ and $\rho \sigma \beta=\sigma \beta$. On the other hand, $G(N / L)=G(N \cdot C(\sigma) / L \cdot C(\sigma))$ by Theorem 2 and $\sigma \beta \in N \cdot \sigma N=N \cdot C(\sigma)$. Thus, we conclude that $\sigma \beta$ is an invariant of $G(N \cdot C(\sigma) / L \cdot C(\sigma))$ in $N \cdot C(\sigma)$. Therefore, $\sigma \beta \in L \cdot C(\sigma)$ and $\sigma L \subset L \cdot C(\sigma) \subset L U_{c}$. Hence, (d) is satisfied.

Finally, assume that (d) is satisfied. Let $\tau$ be any differential isomorphism of $L$ over $K$. The fact that $N$ is finitely $L$-separable and Lemma 1 imply that $\tau$ can be extended to an element $\sigma \in G$. Thus, 
we have $\tau L=\sigma L \subset L U_{c}$. By Proposition 10, we conclude that $L$ is strongly normal over $K$, i.e. (a) is satisfied.

We have proved that the four conditions are mutually equivalent. Now, assume that these conditions are satisfied. It is clear that, in the sequence (3), $\varphi$ is a group-homomorphism and $\operatorname{ker} \varphi=\operatorname{im} l$. The fact that $N$ is finitely $L$-separable and Lemma 1 imply that $\varphi$ is surjective. It remains to prove that $\varphi$ is a $C$-homomorphism.

Let $\sigma, \tau \in G$. We must verify the following three properties.

$1^{\circ} . C(\sigma)=N \cdot \sigma N \cap U_{c} \supset L \cdot \varphi(\sigma) L \cap U_{c}=C(\varphi(\sigma))$.

$2^{\circ}$. Assume $\sigma \rightarrow \tau$. Since $(\tau \alpha ; \alpha \in N)$ is a differential specialization of $(\sigma \alpha ; \alpha \in N)$ over $N,(\varphi(\tau) \beta ; \beta \in L)$ is a differential specialization of $(\varphi(\sigma) \beta ; \beta \in L)$ over $L$, i.e. $\varphi(\sigma) \rightarrow \varphi(\tau)$.

$3^{\circ}$. Assume $\sigma \leftrightarrow \tau$. By $2^{\circ}$, we have $\varphi(\sigma) \leftrightarrow \varphi(\tau)$. The induced isomorphism $S_{\tau, \sigma}: C(\sigma) \rightarrow C(\tau)$ is the restriction of the differential isomorphism $N \cdot \sigma N \rightarrow N \cdot \tau N$ over $N$ which is determined by $\sigma \alpha \rightarrow \tau \alpha(\alpha \in N)$. Considering every element $\alpha$ of $L$, we see that $S_{\varphi(\tau), \varphi(\sigma)}: C(\varphi(\sigma)) \rightarrow C(\varphi(\tau))$ is a restriction of $S_{\tau, \sigma}$.

5. Examples. At first, we provide a well-known example of strongly normal extension.

EXAMPle 1. (See [2]-III-5, IV-5 and VI-1.) Let $X$ be a differential indeterminate over $K$, let $\Lambda$ be a set of linear differential forms in $X$ over $K$ of finite order $n$ and let $\left(x_{1}, \ldots, x_{n}\right)$ be a fundamental system of zeros of $\Lambda$ in a differential extension field of $K$. Set $N=K\left\langle x_{1}, \ldots, x_{n}\right\rangle$. If $N_{c}=K_{c}$ and $N$ is separable over $K$, then we call $N$ a Picard-Vessiot extension of $K$. Then, we can see that $N$ is a strongly normal extension of $K$ and the Galois group $G(N / K)$ is identified with a subgroup of the general linear group $\mathrm{GL}(n)$ relative to the universal field $U_{c}$. Thus, $G(N / K)$ is a linear $C$-group in the sense of [1]-V.

The following example demonstrates the necessity of the remark to Theorem 3.

EXAMPLE 2. Let $K$ be a differential field with a single derivation $\delta=\left(\delta_{\nu} ; \nu=0,1,2, \ldots\right)$. Assume that $\delta$ is trivial in $K$. Choose elements $a_{\nu}(\nu=1,2, \ldots)$ in $K$ satisfying the following conditions:

(a) $a_{\nu}=0$ if $\nu \neq p(e)$ for every natural number $e$.

(b) The number of $\nu$ such that $a_{\nu} \neq 0$ is finite and nonzero.

Let $\mathfrak{p}$ be the differential prime ideal generated by $\delta_{\nu} X-a_{\nu}(\nu=$ $1,2, \ldots)$ in $K\{X\}$. There exists a generic zero $x$ of $\mathfrak{p}$ over $K$. We can see that $N=K\langle x\rangle=K(x)$ is strongly normal over $K$; moreover, 
$N$ is a Picard-Vessiot extension of $K$. Now, assume that $N_{c}$ is not perfect. There is an element $c \in N_{c}$ such that $c^{1 / p} \notin N_{c}$. Set $M=$ $K\left(x^{p(3)}-c^{p} x^{p(2)}\right)$ and $L=K\left(x^{p(2)}-c x^{p}\right)$. Then, $L$ is the purely inseparably algebraic closure in $N$ of $M$. The element $x$ of $N$ is not separable over $L$, since the minimal polynomial of $x$ over $L$ is $X^{p(3)}-c^{p} X^{p(2)}-a$, where $a=x^{p(2)}-c x^{p} \in L$. Hence, $N$ is not $L$-separable and $N$ is not strongly normal over $L$. Since $c$ is constant, $\delta$ can be extended to $c^{1 / p}$. The minimal polynomial of $x$ over $L_{\infty}$ is $X^{p(2)}-c X^{p}-b$, where $b=x^{p}-c^{1 / p} x \in L_{\infty}$, and $x$ is separable over $L_{\infty}$. Hence $N L_{\infty}$ is strongly normal over $L_{\infty}$ and the field of constants of $N L_{\infty}$ is the perfect field $C_{i}$.

Some more examples of the Picard-Vessiot extension by Okugawa can be found in [2] together with several computations of differential Galois groups.

Acknowledgments. The author wishes to express her sincere gratitude to Professor Kôtaro Okugawa for his kind advice. The author wishes also to thank the referee for valuable criticisms which helped greatly to improve the paper.

\section{REFERENCES}

[1] E. R. Kolchin, Differential Algebra and Algebraic Groups, Academic Press, 1973.

[2] K. Okugawa, Differential algebra of nonzero characteristic, Lectures in Mathematics, Department of Math., Kyoto Univ., 16 (1987).

[3] B Basic properties of differential fields of an arbitrary characteristic and the Picard-Vessiot theory, J. Math. Kyoto Univ., 2 (1963), 296-322.

[4] K. Shikishima, Maximal differential field and its applications, Acta Hum. Sci., Univ. Sangio Kyotiensis, IX, Nat. Sci. Ser. (1980), 1-10.

[5] K. Tsuji (nee Shikishima), Differential closure of differential field of positive characteristic, J. Math. Kyoto Univ., 26-4 (1986), 711-715.

[6] A. Weil, Foundations of algebraic geometry, Revised and enlarged ed., Amer. Math. Soc. Colloq. Publ., XXIX (1962).

Received July 13, 1987 and in revised form May 6, 1988.

8-E4-401,

OTOKOYAMA-YUTOKU,

YAWATASHI, 614 JAPAN 